\title{
O Complexo Teniase Humana-Cisticercose: ainda um sério problema de saúde pública
}

\author{
Revisão de literatura
}

\section{The Complex Human Taeniasis-Cysticercosis}

\author{
Literature Review
}

\section{Resumo}

O presente trabalho tem como objetivo revisar o complexo teníase-cisticercose. Trata-se de um sério problema de saúde pública, representado por entidades zoonóticas das mais importantes na atualidade. O complexo compreende duas doenças distintas, com sintomatologia e epidemiologia totalmente diferentes: a teníase que corresponde à fase final do ciclo do parasita e ocorre apenas no ser humano e a cisticercose que corresponde ao estágio larval da Taenia saginata, a qual parasita bovinos, ou da Taenia solium, que pode acometer suínos e também seres humanos. A cisticercose e a teníase são encontradas com maior frequência em países cujas populações apresentam hábitos de higiene precários e com saneamento básico deficiente. Sua etiologia inclui a Taenia solium e a Taenia saginata que pertencem à classe Cestoidea, ordem Cyclophillidea, família Taenidae e gênero Taenia e as respectivas formas larvares, Cysticercus cellulosae e Cysticercus bovis. Na teníase a sintomatologia clínica é bastante variável, de acordo com a idade e o grau de higidez orgânica do hospedeiro. Em condições naturais, os bovinos acometidos por cisticercose não manifestam sinais clínicos, já na cisticercose humana, variam dependendo da localização dos cisticercos. Quando localizados no sistema nervoso central (neurocisticercose) os sinais clínicos podem variar de acordo com o número de cisticercos, seu estado de desenvolvimento, a variedade morfológica, com sua localização e com as reações que provocam no paciente. O controle da teníase-cisticercose depende das condições econômicas, sociais e culturais de cada região e país, tendo a educação sanitária como ferramenta fundamental.

\section{Summary}

This paper aims at reviewing the taeniasis-cysticercosis complex disease. It is a disease that represents a serious public health problem, thus becoming one of the most important zoonotic diseases. The complex comprises two distinct diseases, with totally different symptoms and epidemiology: taeniasis that corresponds to the final stage of the parasite cycle and occurs only in humans and cysticercosis that is the larval stage of Taenia saginata, which is a cattle parasite, and also Taenia solium, which can also affect pigs and humans. Cysticercosis and taeniasis are found more frequently in countries whose populations have poor hygiene and poor sanitation. The taeniasis-cysticercosis complex is formed by Taenia solium and Taenia saginata of Cestoda class, Cyclophillidea order, family and gender Taenidae Taenia and their larval forms, Cysticercus cellulosae and Cysticercus bovis. Taeniasis, in its clinical symptoms, is highly variable, according to the age and health status of the host. Under natural conditions, animals affected by cysticercosis do not show any clinical signs. On the other hand, human cysticercosis may vary depending on the location of cysticerci. When located in the central nervous system (neurocysticercosis) clinical signs can vary depending on the number of cysticerci, their development stage, morphological variety, its location and the immune response they might cause in the patient. The control of taeniasis-cysticercosis depends on the economic, social and cultural needs of each region and country, and health education is a fundamental tool. 
Naassom Almeida Souza Ribeiro ${ }^{1}$ Evelise Oliveira Telles ${ }^{2}$ Simone de Carvalho Balian ${ }^{3}$
Avenida Gustavo Adolfo, 2831

São Paulo-SP, Brasil - 02209-001

\nassaribeiro@hotmail.com

\section{Introdução e Revisão de Literatura}

O complexo teníase-cisticercose pode ser conceituado como um conjunto de patologias decorrentes da infestação/infecção de hospedeiros suscetíveis às formas adultas da Taenia solium e da Taenia saginata e seus respectivos estágios larvares representando sério problema de saúde pública por constituir-se em um dos mais importantes grupos de zoonoses na atualidade. Este agregado conjuntural compreende entidades nosológicas distintas com sintomatologia e epidemiologia totalmente diferentes: i) as teníases que decorrem da infestação do ser humano, pelo estágio adulto, compreendendo a fase final do ciclo das tênias e; ii) as cisticercoses, decorrentes da infecção pelos correspondentes estágios larvares, seja da Taenia saginata, que acomete bovinos, ou da Taenia solium, que pode acometer suínos e também seres humanos (FALAVIFNA-GUILHERME et al., 2006; CHAGAS et al., 2009; GANC et al., 2009; MARQUES; SEIDEL; FIORAVANZO, 2009).

"A importância do complexo está descrita em vários trabalhos sobre o tema, citando os prejuízos que acarretam ao rebanho bovino e suíno, causados pelas condenações de vísceras e carcaças. Das consequências funestas à saúde humana, ressalta a neurocisticercose e sua grande representatividade entre as patologias inflamatórias do sistema nervoso central, que pode levar à morte e a cisticercose intraocular, que pode levar a cegueira" (AGRODEFESA, 2011).

1 Médico Veterinário, CRMV-SP n 22922, Doutorando pela Faculdade de Medicina Veterinária e Zootecnia da Universidade de São Paulo

2 Professora Associada da Faculdade de Medicina Veterinária e Zootecnia da Universidade de São Paulo

3 Professora Doutora da Faculdade de Medicina Veterinária e Zootecnia da Universidade de São Paulo 
Ungar (1986), Fukuda (2003), Pires (2008) e Ganc et al. (2009), referem que as cisticercoses e as teníases são encontradas com maior frequência em países cujas populações apresentam hábitos de higiene precários e deficientes sistemas de saneamento básico, sendo, rara na Europa e nos Estados Unidos. A América Latina constitui a área de ampla distribuição geográfica de ocorrência das cisticercoses, sendo referida desde o México até a Argentina e Chile. No Brasil, têm sido mais estudadas no estado de São Paulo, onde ainda são bastante frequentes.

\section{Etiologia}

Os agentes etiológicos das teníases do presente complexo são representados pela Taenia solium e pela Taenia saginata que pertencem à classe Cestoidea, ordem Cyclophillidea, família Taenidae e gênero Taenia; enquanto a etiologia das cisticercoses é constituída pelas respectivas formas larvares, o Cysticercus cellulosae, que acomete suínos e humanos e Cysticercus bovis, que afeta os bovinos. A infecção humana por ovos de T. solium leva à formação de cistos em diversos tecidos, incluindo o Sistema Nervoso (neurocisticercose) e o globo ocular (cisticercose intraocular) com graves repercussões para a saúde humana e, em outras regiões do organismo também preocupantes, do mesmo modo que o parasitismo pelas formas adultas (PIRES, 2008; CHAGAS et al., 2009, AGRODEFESA, 2011).

\section{Ciclo evolutivo}

Participam do ciclo evolutivo da Taenia solium duas espécies hospedeiras: uma para a forma adulta, denominada hospedeiro definitivo, isto é, o ser humano que a alberga no intestino delgado e outra para a forma larvar, que pode ser o suíno doméstico, selvagem e o ser humano. Da mesma forma, a Taenia saginata se utiliza, fundamentalmente, de duas espécies hospedeiras: o homem, que é o hospedeiro definitivo e o bovino, denominado hospedeiro intermediário, pois nele só ocorre a fase larvar, denominada cisticerco (PIRES, 2008) (Figura 1), podendo acometer também mamíferos artiodáctilos, que servem como hospedeiros intermediários (PAN AMERICAN HEALTH ORGANIZATION, 2003). Acha e Szyfres (2002) comentam que a cisticercose humana por ingestão de ovos da Taenia saginata não ocorre ou é extremamente rara. De acordo com a Pan American Health Organization (2003), no periodo de 1993 a 2003, não houve relatos na literatura, de casos de cisticercose humana causada pelo Cysticercus bovis.
Na cisticercose, os bovinos, suínos e o ser humano adquirem a infecção quando ingerem alimentos e água contaminados com ovos dos respectivos parasitos. O ser humano albergando a forma adulta da Taenia solium, pode sofrer também a autoinfecção, quando por precárias práticas de higiene leva à boca, mãos ou objetos contaminados por ovos, após sua própria defecação, ou mesmo por movimentos retrógrados do sistema digestório (refluxos, vômitos). (GANC et.al., 2009; AGRODEFESA, 2011).

A ingestão, pelo ser humano, de carne crua ou mal cozida, de bovinos ou suínos portadores de cisticercos vivos pode levar à eclosão do cisto no estômago, fixação da larva na mucosa intestinal e desenvolvimento da forma adulta da tênia correspondente, em média, à três meses após a ingestão do cisticerco. O verme, já adulto, começa liberar as proglotes (fragmentos do próprio corpo, como anéis) repletos de ovos. Estima-se que cada tênia libere de 1 a 5 anéis por dia, e que cada proglote contenha, em média, 40 mil ovos embrionados, infectivos e altamente resistentes às condições adversas do ambiente, podendo permanecer viáveis por até oito meses, principalmente em locais com clima quente e úmido, características presentes na grande maioria dos países em desenvolvimento da África, Ásia e América Latina, regiões consideradas endêmicas para a doença (ESTEVES, 2005; SANTOS, 2008; GANC et al., 2009; AGROSOFT BRASIL, 2011;).

Um ser humano infestado pode eliminar milhões de ovos livres nas fezes, diariamente, os quais podem sobreviver no ambiente por vários meses (SILVA, 2009; GANC et al., 2009; AGROSOFT BRASIL, 2011).

O hábito pouco higiênico de evacuar a céu aberto, a existência de sanitários sem as devidas fossas, a inexistência de sanitários (como ocorre em extensas áreas de monoculturas agrícolas), sanitários instalados sobre córregos e rios e a prática de criar suínos alimentados com excretas humanas, contribuem para a manutenção do ciclo. Assim, ao defecar em locais abertos, o homem possibilita a dispersão dos ovos pelo ambiente. As fezes ao serem expostas ao sol secam e possibilitam que os ovos da tênia tornem-se mais leves que as partículas de pó, sendo lançados a grandes distâncias pelo vento, o que pode levar à contaminação de rios, plantações, lagoas etc. A água contaminada pode ser utilizada na dessedentação animal, na irrigação de hortas e para o consumo humano, contribuindo para a transmissão da cisticercose.

Assim, vários autores (PIRES, 2008; GANC et al., 2009; AGROSOFT BRASIL, 2011) concordam que: não é o suíno que infecta o ser humano e sim o ser humano que transmite a doença para o suíno. Por muitos 
anos, no contexto das ciências da saúde, julgava-se o suíno o agente determinante da ocorrência da doença no ser humano. No contexto dos complexos teníases humanas-cisticercoses, os fatores determinantes da doença compreendem falta de saneamento básico, precárias práticas de higiene pessoal, falta de diagnóstico e tratamento dos portadores de tenias e o acesso dos bovinos e suínos aos excrementos humanos, à água e aos vegetais contaminados.

No que se refere ao ciclo ser humano-bovino, vale ressaltar a intensificação da agricultura, com vastas áreas de monoculturas, como por exemplo, extensas áreas de plantio de cana-de-açúcar em São Paulo, exigindo a concentração de lavradores nos campos, onde não há infraestrutura sanitária para as práticas fisiológicas de excreção de fezes e urina. Na possibilidade da existência de lavradores portadores da tênia, ao defecar nos ambientes rurais abertos, basta o vento e a chuva para disseminar nas pastagens ovos infectantes, que acometerão bovinos em fase de engorda nos sistemas extensivos e semiextensivos e, mais uma vez, se tem o ciclo estabelecido.

Quando bovinos e/ou suínos ingerem, direta ou indiretamente, esses ovos, adquirem a cisticercose (PIRES, 2008). A ingestão de ovos pelos animais ocorre, na maior parte das vezes, por ingestão de fezes. Bovinos ingerem fezes somente em condições adversas (falta de alimento), já os suínos, possuidores de hábitos coprofágicos, têm mais facilidade de adquirir a doença (PIRES, 2008) quando criados em condições adversas e totalmente insalubres.

Vollkopfy (2008) e Ganc et al. (2009), referem que a principal forma de ocorrer cisticercose em humanos é a ingestão do ovo da tênia em produtos hortifrutigranjeiros contaminados que sofrem manipulação inadequada ou devido aos maus hábitos de higiene.

O consumo de verduras cruas pode ser uma prática muito favorável para a transmissão de doenças parasitárias, pois ainda existe, não sendo rara, a prática de irrigar hortas com água contaminada com material fecal humano ou, ainda, utilizar dejetos humanos como adubo, principalmente em regiões com baixo índice de desenvolvimento socioeconômico. A divulgação ampla e clara da necessidade de implementar boas práticas agrícolas é fundamental para o controle de perigos biológicos de destaque na saúde pública.

O mecanismo de transmissão oral-fecal ocorre muito frequentemente nas doenças veiculadas por alimentos. Pires (2008) afirma que no caso da cisticercose a disseminação direta é a forma mais relevante para a ocorrência da doença, em detrimento da dispersão natural dos ovos pelo ambiente, por meio do vento ou de água contaminada.

\section{Sintomas clínicos}

\section{Teníase Humana}

$\mathrm{Na}$ teníase humana a sintomatologia clínica é bastante variável, de acordo com a idade e o grau de higidez orgânica do hospedeiro. São registrados sintomas como irritabilidade, insônia, anorexia ou apetite exagerado, perda de peso, dor abdominal, distúrbios digestivos, náuseas, vômitos, diarréia alternada com constipação, perturbações nervosas, fraqueza muscular, sensação de dor e de fome. Muitas infestações são assintomáticas, exceto pelo incômodo de ter segmento de vermes emergindo pelo ânus. Os sintomas característicos da doença, como dores abdominais, náuseas, debilidade, perda de peso, flatulência, diarréia ou constipação, raramente estão presentes. Na maioria dos casos o paciente percebe alguma alteração quando da observação da liberação das proglotes, fato este, que pode ocorrer vários meses após a infestação. Essa é uma característica da relação hospedeiro-parasita muito importante epidemiologicamente, pois o acometido poderá disseminar ovos para o ambiente durante longos períodos, antes mesmo de ter sido diagnosticado (GANC et al., 2009; MARQUES; SEIDEL; FIORAVANZO, 2009).

\section{Cisticercose animal}

Em condições naturais, os bovinos acometidos por cisticercose não manifestam sinais clínicos. Entretanto, experimentalmente, bezerros submetidos à infecções maciças por ovos de Taenia saginata desenvolveram grave miocardite e insuficiência cardíaca associadas aos cisticercos em desenvolvimento no coração (SILVA, 2009). Essa informação reforça, mais uma vez, a importância da inspeção rigorosa do coração durante práticas de abate, sendo aconselhável posterior reinspeção e monitoramento.

No suíno infectado pode-se observar, em casos isolados, hipersensibilidade no focinho, paralisia da língua e convulsões epileptiformes, porém, a brevidade da vida dos suínos impede a observação de manifestações neurológicas (PIRES, 2008).

Silva (2009) considera que durante a fase de disseminação, os sintomas, quando presentes, estão relacionados com a distribuição dos embriões nos diferentes tecidos e nesses casos, pode ser observada dificuldade na apreensão de alimentos, mastigação e, até mesmo, pseudoparalisia do maxilar inferior, no caso de infecção maciça dos músculos mastigadores e da língua e tosse seca e quitinosa quando localizados nos músculos ou na submucosa da laringe, além de transtornos cerebrais em casos de infecções severas.

\section{Cisticercose humana}

A Organização Mundial da Saúde (WHO, 200o), Takayanagui e Leite (2001) e ABIPECS (2009), referem 
que a neurocisticercose é responsável por 50.000 óbitos por ano em países em desenvolvimento, principalmente da Ásia, África e América Latina. No Brasil, os Estados de São Paulo, Minas Gerais, Paraná e Goiás apresentam a doença em forma endêmica (MENDES, 2005).

Os sinais clínicos variam dependendo da localização dos cisticercos. Quando localizados no sistema nervoso central (neurocisticercose), os sinais clínicos podem variar de acordo com o número de cisticercos, seu estado de desenvolvimento, variedade morfológica e com sua localização. Os sintomas mais frequentes são: crises convulsivas, hipertensão intracraniana, hidrocefalia e distúrbios psiquiátricos. Os cisticercos podem também se alojar em outras estruturas, como coração, olhos e músculos (TAKAYANAGUI e LEITE, 2001; ABIPECS, 2009).

A localização ocular causa redução da visão, irites, uveites, retinites, moscas volantes, exoftalmia ou miosite com ptose e conjuntivites. O prognóstico visual dos portadores de cisticercose intraocular permanece incerto, face aos efeitos danosos produzidos pela presença do parasito e, mais acentuadamente, após a sua desintegração no interior do globo ocular (PIRES, 2008).

Hidalgo (2008) relatou o registro de 937 óbitos por cisticercose no Brasil, no período de 1980 a 1898.

Santo (2007), afirmou que a mortalidade relacionada à cisticercose na população não tem sido pesquisada no Brasil; o autor estudou os óbitos registrados entre 1985 e 2004 no Estado de São Paulo em que a cisticercose foi mencionada em qualquer linha ou parte da Declaração de Óbito. As causas de morte foram processadas pelo Tabulador de Causas Múltiplas. No período de vinte anos ocorreram 1.570 óbitos, sendo a cisticercose causa básica em 1.131 e causa associada de morte, em 439 casos. Os coeficientes padronizados de mortalidade por cisticercose como causa básica apresentaram tendência de declínio, maiores entre os homens e nas idades mais avançadas.

As principais causas associadas às mortes por cisticercose foram a hipertensão intracraniana, o edema cerebral, as hidrocefalias, as doenças inflamatórias do sistema nervoso central e as doenças cerebrovasculares (SANTO, 2007).

A Síndrome da Imunodeficiência Adquirida (SIDA) foi a principal causa básica nas mortes, tendo como causa associada, a cisticercose. Os municípios com maior número de mortes foram São Paulo, Campinas, Ribeirão Preto e Santo André. Observou-se grande variação entre os municípios na valorização da cisticercose como causa básica de morte, determinando sua subestimada importância para subsidiar o planejamento de políticas de saúde (SANTO, 2007).

\section{Controle e Prevenção}

Germano e Germano (2003) e Esteves et al. (2005) e para a Canadian Food Inspection Agency (CFIA) (2011), destacam que o controle da teníase-cisticercose depende das condições econômicas, sociais e culturais em cada região e país. Serêa et al. (2006), indicam a educação sanitária como ferramenta fundamental no controle do complexo teníase-cisticercose.

Almeida et al. (2006) e Souza et al. (2007), referem quem a inspeção veterinária de carnes, executada pelo Serviço de Inspeção Federal (SIF), é a medida direta de maior importância na prevenção da teníase, pois apesar de suas limitações, a inspeção identifica as carcaças com infecções intensas e leves, desde que exista alguma alteração visível macroscopicamente, e atua também como crivo sanitário, impedindo a disseminação de agentes zoonóticos.

Vale lembrar que todos os estabelecimentos que realizam práticas de abate de animais de corte, obrigatoriamente, devem realizar a inspeção ante e post mortem dos animais e de suas carcaças e vísceras (ARIISPO, 2008), sejam eles fiscalizados por Serviços de Inspeção Federal, Estadual ou Municipal (AGRODEFESA, 2011).

\footnotetext{
"A inspeção sanitária específica para a pesquisa de cisticercos fundamenta-se na avaliação visual, na palpação e em cortes dos músculos da cabeça, língua, coração, diafragma, músculos do pescoço e intercostais. A liberação da carcaça para consumo "in natura" dá-se quando da ausência de cisticercos. Quando da presença de cisticerco será dado aproveitamento condicional ou condenação total, dependendo do número de cisticercos e de seu estado de desenvolvimento e vitalidade" (AGRODESEFA, 2011).

\begin{abstract}
"Os relatórios sobre as ocorrências são enviados para a Agrodefesa que através dos técnicos lotados nas unidades locais que fazem visitas às propriedades a fim de investigar o foco, indicar tratamento dos animais, manejo da criação e é realizado em parceira com a Secretaria da Saúde o trabalho de Educação Sanitária" (AGRODESEFA, 2011).
\end{abstract}

No Brasil, ocorrem duas situações distintas para os complexos teníase humana-cisticercose bovina e suína, respectivamente. No contexto do complexo Taenia saginata/Cysticercus bovis a manutenção do ciclo é determinada pela ausência de saneamento básico em áreas rurais, fundamentalmente dedicadas à bovinocultura de corte, enquanto que no contexto da teníase/cisticercose por Taenia sollium/Cysticercus cellulosae o ciclo se perpetua a partir de produções de suínos em condições 
precárias de higiene, tecnologia e sanidade, alimentados com resíduos orgânicos, em convívio direto com seres humanos e abatidos sem inspeção veterinária. Conclui-se que o seu controle depende, fundamentalmente, das condições econômicas, sociais, culturais e políticas de cada região e país, tendo a educação sanitária como ferramenta fundamental.

\section{Referências}

1. ABIPECS - Associação Brasileira da Indústria Produtora e Exportadora de Carne Suína. Carne suína brasileira. Disponível em: <http://www.carnesuinabrasileira.org.br/ medicina2.html> Acesso em: 23 nov. 2009.

2. ACHA, P.N.; SZYFRES, B. Zoonosis y enfermidades transmissibles comunes al hombre y a los animales. 3. ed. Washington: OMS, 2001-2002.

3. AGRODEFESA. Programa Estadual de Controle do Complexo Teníase Cisticercose Disponível em: <http://www.agrodefesa.go.gov.br/index.php?option=com_conte $n t \& v i e w=$ article\&id=69\&ltemid=11>. Acesso em: 21 out. 2011.

4. AGROSOFT BRASIL. Conhecendo a prevalência da cisticercose suína e bovina no Brasil: devemos rever nossos hábitos alimentares? [2006]. Disponível em: <http:// www.agrosoft.org.br/agropag/19197.htm>. Acesso em: 21 out. 2011.

5. ALMEIDA, D.O.; IGREJA, H.P.; ALVES, F.M.X.; SANTOS, I.F.; TORTELLY, R. Cisticercose bovina em matadouro-frigorífico sob inspeção sanitária no município de Teixeira de Freitas-BA: prevalência da enfermidade e análise anatomopatológica de diagnósticos sugestivos de cisticercose. Revista Brasileira Ciências Veterinárias, v. 13 , n. 3, p. $178-182$, set./dez. 2006

6. CFIA - CANADIAN FOOD INSPECTION AGENCY. Bovine Cysticercosis. [2009]. Disponível em: <http://www.inspection.gc.ca/english/anima/disemala/ rep/2009bovinecyste.shtml>. Acesso em: 21 out. 2011.

7. CHAGAS, L.D.S.; LOPES. E.F.; MAANZANI, N.H.; SANTOS, M.C.D.; NASCIMENTO, A.F.; OLIVEIRA, L.S.R.; ALMEIDA, L.P. O complexo teníase-cisticercose em pequenas propriedades rurais de Uberlândia - MG. 2008. Disponível em: <http:// www.ic ufu.org/anaisufu2008/PDF/SA08-11038.PDF>. Acesso em: 17 nov. 2009.

8. ESTEVES, F.M.; SILVA-VERGARA, M.L.; CARVALHO, A.C.F.B. Inquérito epidemiológico sobre teníase em população do programa saúde da família no município de Uberaba, MG. Revista Sociedade Brasileira Medicina Tropical, v.38, p.530-531, 2005.

9. FALAVIGNA-GUILHERME, A.L.; SILVA, K.; ARAÚJO, S.M.; TOBIAS, M.L.; FALAVIGNA, D.L.M. Cisticercose em animais abatidos em Sabáudia, Estado do Paraná. Arquivo Brasileiro de Medicina Veterinaria e Zootecnia, Belo Horizonte, v. 58, n. 5, 2006.

10. FUKUDA, R.T. Contribuição ao estudo da epidemiologia da cisticercose bovina na região administrativa de Barretos. Aspectos ambientais e econômicos. 2003. 127 f. Tese (Doutorado em Medicina Veterinária) - Faculdade de Ciências Agrárias e Veterinárias, Universidade Estadual Paulista, Jaboticabal, 2003

11. GANC, A.J.; CORTEZ, T.L.; VELOSO, PP.A. A carne suína e suas implicações no complexo teníase-cisticercose. Disponível em: <http://www.abcs.org.br/portal/ mun_car/medico/artigos/4.pdf>. Acesso em: 19 jul. 2009.
12. GERMANO, P.M.L.; GERMANO, M.I.S. Higiene e vigilância sanitária de alimentos 2. ed. São Paulo: Varela, 2003. p.335-337.

13. HIDALGO, S.C.F. Panorama da cisticercose no Brasil. 2008, 47 f. Monografia - Instituo Brasileiro de Pós Graduação Qualittas Ltda, Campo Grande, 2008.

14. MARQUES, S.M.T.; SEIDEL, E.; FIORAVANZO, R.F. Complexo Teníase/Cisticercose: uma parasitose emergente. 2008. Disponível em: <http://www.sovergs.com.br/ conbravet2008/anais/cd/resumos/R1048-2.pdf>. Acesso em: 17 nov. 2009.

15. MENDES, E.C.; SILVA, S.S.; FONSECA, E.A.L.T.; SOUZA, H.R.R.; CARVALHO, R.W. A Neurocisticercose Humana na Baixada Fluminense, Estado do Rio de Janeiro, Brasil. Rev. Arquivo Neuropsiquiatria, v. 63, n.4, p.1058-1062, 2005.

16. PAN AMERICAN HEALTH ORGANIZATION. Zoonoses and Cammunicable diseases common to man and animals. Third Edition. 3rd. ed. Washington, D.C.: PAHO, 2003. 3 vol. - (Scientific and Technical Publication $N^{\circ} .580$ ). p. 166

17. PIRES, W.M. Complexo teníase-cisticercose. 2008. 25 f. Monografia (Especialização Lato sensu em Higiene e Inspeção de Produtos de Origem Animal) - Instituto Qualittas de Pos-Graduação, Palmas, 2008.

18. SANTO, A.H. Cysticercosis-related mortality in the State of São Paulo, Brazil, 19852004: a study using multiple causes of death. Caderno Saúde Pública, Rio de Janeiro, v. 23, n. 12,2007

19. SANTOS, J.P. Prevalência de cisticercose bovina em matadouro - frigorífico sob inspeção estadual (SIE) em Santa Catarina. 2008. 37 f. Monografia (Pós-Graduação em Higiene e Inspeção de Produtos de Origem Animal - Vigilância Sanitária) - Universidade Castelo Branco, Curitiba, 2008.

20. SERÊA, T.G.; TOBIAS, M.L.; FALAVIGNS, D.L.M.; FALAVIGNA-GUILHERME, A.L. Educação sanitária como ferramenta no controle do complexo teníase-cisticercose. Trabalho apresentado no IV Fórum de Extensão e Cultura da UEM. Perspectivas da Extensão Universitária e da Prestação de Serviços. Arq. Mudi., v. 10 Suplemento 1. 2006

21. SILVA, A.V. da. Complexo teníase cisticercose. UNIPAR Umuarama, slides, 2007 Disponível em: <http://virtual.unipar.br/courses/DPAD/document/3o_Bimestre/ SlidesCTC2007.pdf?cidReq=DPAD>. Acesso em: 19 jul. 2009.

22. SOUZA, V.K.; SILVA, M.C.P.; MINOZZO, J.C.; THOMAZ-SOCCOL, V. Prevalência da cisticercose bovina no estado do Paraná, sul do Brasil: avaliação de 26.465 bovinos inspecionados no SIF 1710. Semina: Ciências Agrárias, Londrina, v.28, n.4 p.675-684, 2007.

23. TAKAYANAGUI, O.M.; LEITE, J.P. Neurocisticercose. Revista da Sociedade Brasileira de Medicina Tropical, Uberaba, v.34, n.3, 2001.

24. UNGAR, M. L.; GERMANO, M. I. S.; GERMANO, P. M. L. Higiene e vigilância sanitária de alimentos. 3. ed. Barueri: Manole, 2008. $986 \mathrm{p}$

25. VOLLKOPFY, P.V.P. Prevalência de cisticercose em bovinos abatidos sob inspeção sanitária em Campo Grande - MS. 2008. 25 f. Monografia - Universidade Castelo Branco, Campo Grande, 2008.

26. WORLD HEALTH ORGANIZATION - WHO. A Proposal to Declare Neurocysticercosis na International Reportable Disease. Geneva: WHO, 2000. 